\title{
SARS-CoV-2 Prevalence among Outpatients during Community Transmission, Zambia, July 2020
}

Jonas Z. Hines, Sombo Fwoloshi, Davies Kampamba, Danielle T. Barradas, Dabwitso Banda, James E. Zulu, Adam Wolkon, Samuel Yingst, Mary Adetinuke Boyd, Mpanji Siwingwa, Lameck Chirwa, Muzala Kapina, Nyambe Sinyange, Victor Mukonka, Kennedy Malama, Lloyd B. Mulenga, Simon Agolory

During the July 2020 first wave of severe acute respiratory syndrome coronavirus 2 in Zambia, PCR-measured prevalence was $13.4 \%$ among outpatients at health facilities, an absolute difference of $5.7 \%$ compared with prevalence among community members. This finding suggests that facility testing might be an effective strategy during high community transmission.

$\mathrm{T}$ The first cases of infection with severe acute 1 respiratory syndrome coronavirus 2 (SARSCoV-2), the virus that causes coronavirus disease (COVID-19), were reported in Zambia in March 2020 (1). During the first wave of infections, confirmed cases rapidly increased during July and peaked in August 2020 (Appendix, https://wwwnc.cdc.gov/EID/article/27/8/21-0502-App1. pdf). Zambia initially focused on screening travelers at points-of-entry and tracing contacts of persons with laboratory-confirmed cases. In April 2020, the Zambia Ministry of Health began SARSCoV-2 surveillance among hospital inpatients and outpatients to identify cases of local transmission $(1,2)$. It was believed that testing in health facilities would be more efficient at identifying cases than testing in the general population, which was particularly noteworthy given the severe shortage of SARS-CoV-2 tests in Africa early in the pandemic $(3,4)$. A household prevalence survey conducted in

Author affiliations: US Centers for Disease Control and Prevention, Lusaka, Zambia (J.Z. Hines, D.T. Barradas, A. Wolkon, S. Yingst, M.A. Boyd, S. Agolory); University Teaching Hospital, Lusaka (S. Fwoloshi, D. Kampamba, M. Siwingwa, L. Chirwa,

L.B. Mulenga); Zambia Ministry of Health, Lusaka (S. Fwoloshi, K. Malama, L.B. Mulenga); Zambia Field Epidemiology Training Program, Lusaka (D. Banda, J.E. Zulu); Zambia National Public Health Institute, Lusaka (M. Kapina, N. Sinyange, V. Mukonka)

DOI: https://doi.org/10.3201/eid2708.210502
6 districts in Zambia in July 2020 found a community SARS-CoV-2 prevalence of $7.6 \%$ by using realtime PCR (rPCR) (5). To determine if facility testing was an effective case-finding strategy during a period of high community transmission, we compared SARS-CoV-2 prevalence among outpatients, overall and stratified by reasons for visiting the facility, with prevalence among community members in the same districts (5).

\section{The Study}

During July 2-31, 2020, we administered a cross-sectional prevalence survey of patients attending 20 outpatient clinics, including hospitals and health centers, in 6 districts in Zambia (Appendix). The number of facilities we selected in each district was proportional to the number of facilities in the other districts (Appendix). We recruited participants from outpatient departments regardless of their reason for visiting the facilities. Study teams were instructed to recruit $\geq 50$ participants per facility and to attempt to show no preference in selection. We obtained consent or assent (for minors) before beginning study procedures. Participants completed an interviewer-administered questionnaire that included demographics, medical history, SARS-CoV-2 exposures, history of recent illness, and reason for visiting the facility. Concurrently, we conducted a cluster-sampled household prevalence survey in the same 6 districts (5). These surveys provided an opportunity to directly compare outpatient and community SARS-CoV-2 prevalence estimates. The study was approved by the Zambia National Health Research Authority and the University of Zambia Biomedical Research Ethics Committee. The activity was reviewed by the US Centers for Disease Control and Prevention (CDC) and conducted consistent with applicable US federal law and CDC policy. 
We tested nasopharyngeal specimens for SARSCoV-2 RNA by using rPCR and plasma specimens for SARS-CoV-2 antibodies by using ELISA. We extracted RNA for rPCR using the QIAGEN Viral Mini procedure (https://www.qiagen.com). We used the Maccura SARS-CoV-2 Fluorescent PCR kit (https:/ / www.maccura.com) on the QuantStudio 3 platform (ThermoFisher, https://www.thermofisher.com) as the primary rPCR diagnostic (6) and used the CDC assay method to confirm positive and indeterminant results (7). We performed the Euroimmun ELISA (PerkinElmer, https://www.perkinelmer.com) to test for spike protein IgG in single replicate (8). Participants could take part in any or all of the survey, rPCR testing, or serologic testing options based on personal preference.

We calculated SARS-CoV-2 prevalence as the number of positive results divided by the total number of tests conducted. Estimates were calculated separately for rPCR and ELISA results. We adjusted variance and $95 \%$ CIs for clustering by facility and seroprevalence for imperfect assay test characteristics (sensitivity $64.2 \%$; specificity $100 \%$; L. Steinhardt, pers. comm., email, 2021 Apr 2) using the RoganGladen method (Appendix). To assess various factors associated with SARS-CoV-2 prevalence among outpatients, we used bivariate Poisson regression models to calculate prevalence ratios (PRs) and 95\% CIs, with a random effects term for facility.

Of 1,975 persons representing $\approx 3.5 \%$ of $\approx 57,000$ outpatients from the 20 facilities that we approached in July 2020 about participating (District Health Information System version 2; https://dhis2.org), $1,952(98.8 \%)$ completed the questionnaire and 1,908 $(97.7 \%)$ submitted either nasopharyngeal (1,490; $76.3 \%)$ or blood $(1,657 ; 84.3 \%)$ specimens or both (Appendix). Of the 1,952 total participants, the number per district ranged from $160(8.2 \%)$ in Nakonde District to 639 (32.8\%) in Lusaka District; the median number of participants per facility was 93 (interquartile range 78-107; Table 1, https:/ / wwwnc.cdc.gov/
EID/article/27/8/21-0502-App1.pdf). Median participant age was 32 years (interquartile range $24-43$ years); $60.5 \%$ were female. Overall, $34.1 \%$ of participants reported having a coexisting medical condition. Fever or respiratory complaints accounted for $28.2 \%$ of reasons for visiting the facility; $2.3 \%$ of participants were specifically seeking COVID-19 testing.

SARS-CoV-2 rPCR-measured prevalence was $13.4 \%$ (95\% CI 8.3\%-18.5\%; Table 1); SARS-CoV-2 ELISA-measured prevalence was 8.2\% (95\% CI 5.1\%$11.4 \%$ ). Compared with community members, outpatients overall had higher rPCR-measured prevalence (PR 1.8, 95\% CI 1.1-2.9; Table 2) as did those seeking COVID-19 testing (PR 3.6, 95\% CI 2.2-5.9) or those without a stated reason for the visit (PR 2.0, 95\% CI 1.2-3.3). Although only $2.2 \%$ of participants reported contact with confirmed COVID-19 case-patients, rPCR-measured prevalence was higher among outpatients specifically seeking COVID-19 testing compared with outpatients attending facilities for another reason (PR 2.2, 95\% CI 1.4-3.3). In addition, outpatients had higher ELISA-measured prevalence than community members (PR 2.5, 95\% CI 1.4-4.5) (Appendix). Among outpatients with SARS-CoV-2 infection, $45.7 \%$ did not report experiencing any symptoms associated with SARS-CoV-2.

\section{Conclusions}

Outpatients had higher SARS-CoV-2 prevalence than did community members in Zambia. Given the high SARS-CoV-2 prevalence and proportion of asymptomatic infections among outpatients, without instituting protective measures facilities might become transmission foci. Ameliorating risk requires instituting robust prevention and control strategies including universal masking in facilities $(9,10)$. Furthermore, persons seeking testing at facilities should be quickly identified and isolated, because they might be at particularly high risk for having the virus.

One limitation of our study is that underlying condition and exposure history are subject to self-report

\begin{tabular}{|c|c|c|c|}
\hline Population & Prevalence, $\%(95 \% \mathrm{Cl})$ & Prevalence ratio $(95 \% \mathrm{Cl})$ & Absolute difference, $\%(95 \% \mathrm{Cl})$ \\
\hline Community members, $\uparrow n=2,990$ & $7.6(4.7-10.6)$ & Referent & Referent \\
\hline \multicolumn{4}{|l|}{ Outpatients, $n=1,490$} \\
\hline Overall & $13.4(8.3-18.5)$ & $1.8(1.1-2.9)$ & $5.7(0.3-11.2)$ \\
\hline Fever or respiratory complaint & $12.9(6.6-19.2)$ & $1.7(0.9-3.0)$ & $5.3(-1.2$ to 11.7$)$ \\
\hline COVID-19 testing & $27.5(17.7-37.3)$ & $3.6(2.2-5.9)$ & $19.9(10.5-29.3)$ \\
\hline Other acute medical complaints & $10.7(5.6-15.7)$ & $1.4(0.8-2.5)$ & $3.0(-2.4$ to 8.4$)$ \\
\hline Routine health visit & $12.5(4.6-20.3)$ & $1.6(0.8-3.2)$ & $4.8(-2.9$ to 12.5$)$ \\
\hline Not specified & $15.5(9.8-21.2)$ & $2.0(1.2-3.3)$ & $7.9(2.0-13.8)$ \\
\hline
\end{tabular}

${ }^{*}$ COVID-19, coronavirus disease.

†Estimates derived from a cluster-sampled household prevalence survey conducted among community members in the same 6 districts (Kabwe, Livingstone, Lusaka, Nakonde, Ndola, and Solwezi) as in the outpatient prevalence study. 
and recall biases. The districts and facilities were not randomly selected and, despite our intentions to remain unbiased, may not have been representative of the population. The small sample size may have affected our ability to detect differences in factors associated with SARS-CoV-2 prevalence. The higher ELISA-measured prevalence among outpatients than community members could signal noncomparability between these 2 populations or that being an outpatient is a possible marker for other behaviors that increase SARS$\mathrm{CoV}-2$ infection risk. We assumed exact sensitivity and specificity values for the serology assay, but emerging evidence on serologic cross-reactivity (11-13) and antibody decay (14) might affect these values. However, given the timing of our study early in the outbreak, antibody decay was unlikely to substantially affect sensitivity (J. Perez-Saez, unpub. data, https:/ / doi.org/10.1 101/2021.03.16.21253710).

Countries with limited testing capacity need efficient strategies to identify persons with SARS-CoV-2 infections to interrupt transmission. In Zambia, when measured by rPCR, outpatients had $80 \%$ higher SARS$\mathrm{CoV}-2$ prevalence than persons in the surrounding community. Testing all outpatients regardless of their reasons for visiting the facility during periods of community transmission might help identify otherwise undetected SARS-CoV-2 infections. Compared with community-based SARS-CoV-2 testing, outpatient testing, which is often more convenient, might identify cases more effectively. Therefore, testing at facilities during periods of high community transmission might be an effective strategy to identify persons with SARS-CoV-2 infection, especially when testing capacity is limited.

This work has been supported by the President's Emergency Plan for AIDS Relief through the Centers for Disease Control and Prevention and its emergency response to the COVID-19 pandemic.

\section{About the Author}

Dr. Hines is the surveillance advisor in the US Centers for Disease Control and Prevention Center for Global Health field office in Lusaka, Zambia. He is an internal medicine physician with 6 years of international public health experience, including in areas of surveillance methodology and public health workforce capacity building.

\section{References}

1. Chipimo PJ, Barradas DT, Kayeyi N, Zulu PM, Muzala K, Mazaba ML, et al. First 100 persons with COVID-19Zambia, March 18-April 28, 2020. MMWR Morb Mortal
Wkly Rep. 2020;69:1547-8. https://doi.org/10.15585/ mmwr.mm6942a5

2. Zambia Ministry of Health. Implementation strategy - active screening for COVID-19 in health facilities. Lusaka (ZM): Zambia Ministry of Health; 2020.

3. Nkengasong J. Let Africa into the market for COVID-19 diagnostics. Nature. 2020;580:565. https:/ / doi.org/10.1038/ d41586-020-01265-0

4. Kavanagh MM, Erondu NA, Tomori O, Dzau VJ, Okiro EA, Maleche A, et al. Access to lifesaving medical resources for African countries: COVID-19 testing and response, ethics, and politics. Lancet. 2020;395:1735-8. https://doi.org/10.1016/S0140-6736(20)31093-X

5. Mulenga LB, Hines JZ, Fwoloshi S, Chirwa L, Siwingwa M, Yingst S, et al. Prevalence of SARS-CoV-2 in six districts in Zambia in July, 2020: a cross-sectional cluster sample survey. Lancet Glob Health. 2021;9:e773-81. https:/ / doi.org/ 10.1016/S2214-109X(21)00053-X

6. Maccura Biotechnology. SARS-CoV-2 fluorescent PCR kit: instructions for use. 2020 [cited 27 May 2021]. https:/ / www. fda.gov/media/137026/download

7. Centers for Disease Control and Prevention. CDC 2019-novel coronavirus (2019-nCoV) real-time RT-PCR diagnostic panel. 2020 [cited 27 May 2021] https:/ / www.fda.gov/ media/134922/download

8. U.S. Food and Drug Administration. Serology test evaluation report for "SARS-COV-2 ELISA (IgG)" from Euroimmun. 2020 [cited 27 May 2021] https:/ / www.accessdata.fda.gov/ cdrh_docs/presentations/maf/maf3246-a001.pdf

9. Wang X, Ferro EG, Zhou G, Hashimoto D, Bhatt DL. Association between universal masking in a health care system and SARS-CoV-2 positivity among health care workers. JAMA. 2020;324:703-4. https:// doi.org/10.1001/ jama.2020.12897

10. Kim H, Hegde S, LaFiura C, Raghavan M, Sun N, Cheng S, et al. Access to personal protective equipment in exposed healthcare workers and COVID-19 illness, severity, symptoms and duration: a population-based case-control study in six countries. BMJ Glob Health. 2021;6:e004611. https://doi.org/10.1136/bmjgh-2020-004611

11. Tso FY, Lidenge SJ, Peña PB, Clegg AA, Ngowi JR, Mwaiselage J, et al. High prevalence of pre-existing serological cross-reactivity against severe acute respiratory syndrome coronavirus-2 (SARS-CoV-2) in sub-Sahara Africa. Int J Infect Dis. 2020;102:577-83. https:/ / doi.org/ 10.1016/j.ijid.2020.10.104

12. Nkuba Ndaye A, Hoxha A, Madinga J, Mariën J, Peeters M, Leendertz $\mathrm{FH}$, et al. Challenges in interpreting SARS-CoV-2 serological results in African countries. Lancet Glob Health. 2021;9:e588-9. https://doi.org/10.1016/ S2214-109X(21)00060-7

13. Steinhardt LC, Ige F, Iriemenam NC, Greby SM, Hamada Y, Uwandu M, et al. Cross-reactivity of two SARS-CoV-2 serological assays in a malaria-endemic setting. J Clin Microbiol. 2021 Apr 14 [Epub ahead of print]. https://doi.org/10.1128/JCM.00514-21 PMID: 33853839

14. Choe PG, Kim K-H, Kang CK, Suh HJ, Kang E, Lee SY, et al. Antibody responses 8 months after asymptomatic or mild SARS-CoV-2 infection. Emerg Infect Dis. 2021;27:928-31. https://doi.org/10.3201/eid2703.204543

Address for correspondence: Jonas Z. Hines, CDC-Zambia Office, PO Box 320065, 351 Independence Way, Woodlands, Lusaka,

Zambia; email: jhines1@cdc.gov 